\title{
Acute adrenal insufficiency as a fist sign of metastatic pulmonary carcinoma
}

Adina Manolachie ${ }^{1}$, Constantin Volovat ${ }^{2}$, Cristina Grigorescu ${ }^{3}$, Gina Bodnariu ${ }^{4}$, Bogdan Gafton ${ }^{5}$, Ioana Armasu ${ }^{1}$, Letitia Leustean $^{1}$, Carmen Vulpoi $^{1}$ 1-Department of Endocrinology, University of Medicine and Pharmacy 'Gr.T.Popa', Iasi; 2- Department of Oncology, Victoria Hospital, Iasi; 3- Department of Thoracic Surgery, University of Medicine and Pharmacy 'Gr.T.Popa', Iasi; 4- Department of Diabetes, nutrition and metabolic deseases, University of Medicine and Pharmacy 'Gr.T.Popa', Iasi , 5 - Department of Oncology, Regional Institute of Onclolgy, Iasi.

\section{INTRODUCTION}

Adrenal insufficiency:

a relatively rare desease

incidence - new cases $\sim 0.8$ cases 100.000 population per year

Prevalence: $\sim 4-11$ cases i/100.000 of population

- the tuberculous form is most often seen in man : M/F $1.25 / 1$ and the autoiumune in

women F/M ratio 2.6

60-70\% of cases ar diagnosticated between $30-50$ de ani years of age.

ethyology: - most frequent autoimunity or infectious;

sometimes caused by metastatic lesions, genetic disorders and bilateral adrenal haemorrhage.
The adrenal glands are a common sites for secondary lesions derived from malignant melanoma lymphoma, renal, breast, colon and bronchopulmonary tumours.

Adrenal metastasis, at the initial diagnosis of non-small cell lung cancer, occurs in less than $10 \%$ of lung cancer patients.

Most cases involve solitary, unilateral, small asymptomatic lesions.

Bilateral adrenal metastases are observed in less than $3 \%$ of patients with lung cancer.

\section{CLINICAL CASES}

Pacient $\mathrm{C} \mathrm{L}$, 65 years old

Evaluated in the Emergency Department for acute episodes of :

$\begin{array}{cc}\cdot \text { severe asthenia } & \\ \cdot \text { anorexia } & \cdot \text { hyponatremia } 125 \mathrm{mmol} / \mathrm{I} \\ \text { - digestive disorders } & \text { with } \\ \cdot \text { - weight loss } & \text { - hyperkalemia } 5.3 \mathrm{mmol} / \mathrm{l}\end{array}$

no remission of symptoms after administration of macromolecular solutions raised the suspicion of primary adrenal insufficiency, confirmed by functional corticotrop balance: increased ACTH $=344 \mathrm{pg} / \mathrm{ml}$ and

low-normal cortisol levels $=6.38 \mathrm{ug} / \mathrm{dl}$

Steroid replacement (hydrocortizon $15 \mathrm{mg} /$ per day) determined significantly clinical improvement normalization of blood pressure and electrolyte imbalances.

In tha Endocrinology Clinic he presented with: - the absence of melanodermia

- low-normal cortisol levels $=8.02 \mathrm{ug} / \mathrm{dl}$.

- ACTH levels $=101 \mathrm{pg} / \mathrm{ml}$ which increased after

oral steroid replacement was stopped for 48 hours $-465 \mathrm{pg} / \mathrm{m}$.

* Further investigations included abdominal ultrasonography (fig. 1):

$>$ discreet enlargement of the left adrenal $(21 / 20.7 \mathrm{~mm})$

- echogenic lesion on the right adrenal $(29 / 20 \mathrm{~mm})$

$>$ well defined hepatic nodule $(22 \mathrm{~mm})$.

Abdominal CT with contrast (fig. 2):

bilateral adrenal hyperplasia ( $R: 43 / 22 / 47 \mathrm{~mm}, \mathrm{~L}: 33 / 24 / 48 \mathrm{~mm}$ )

observation of hepatic secondary lesion $(6-11 \mathrm{~mm})$ and the biopsy suggested metastasis of lung adenocarcinoma.

\begin{tabular}{|l||l|}
\multicolumn{1}{|c|}{ Microscopy: } & $\begin{array}{l}\text { Immunohistochemistry: } \\
\text { - synaptofizin negative }\end{array}$ \\
- Adenocarcioma metastasis & $\begin{array}{l}\text { - chromogranin negative } \\
\text { - Mildly differentiated }\end{array}$ \\
- CK rarely positive \\
\hline - TTF1 strongly positive
\end{tabular}

* Native chest CT (Figure 3) reveled:

irregular nodular lesion $(11 / 21 / 10 \mathrm{~mm})$ near the oblique fissure.

medistinale lymph nodes (10-22 $\mathrm{mm}$.

* TNM classification: $c$ T1bN2M1bG2

* Chemotherapy was initiated (carboplatin and paclitaxel), well tolerated with favorable evolution

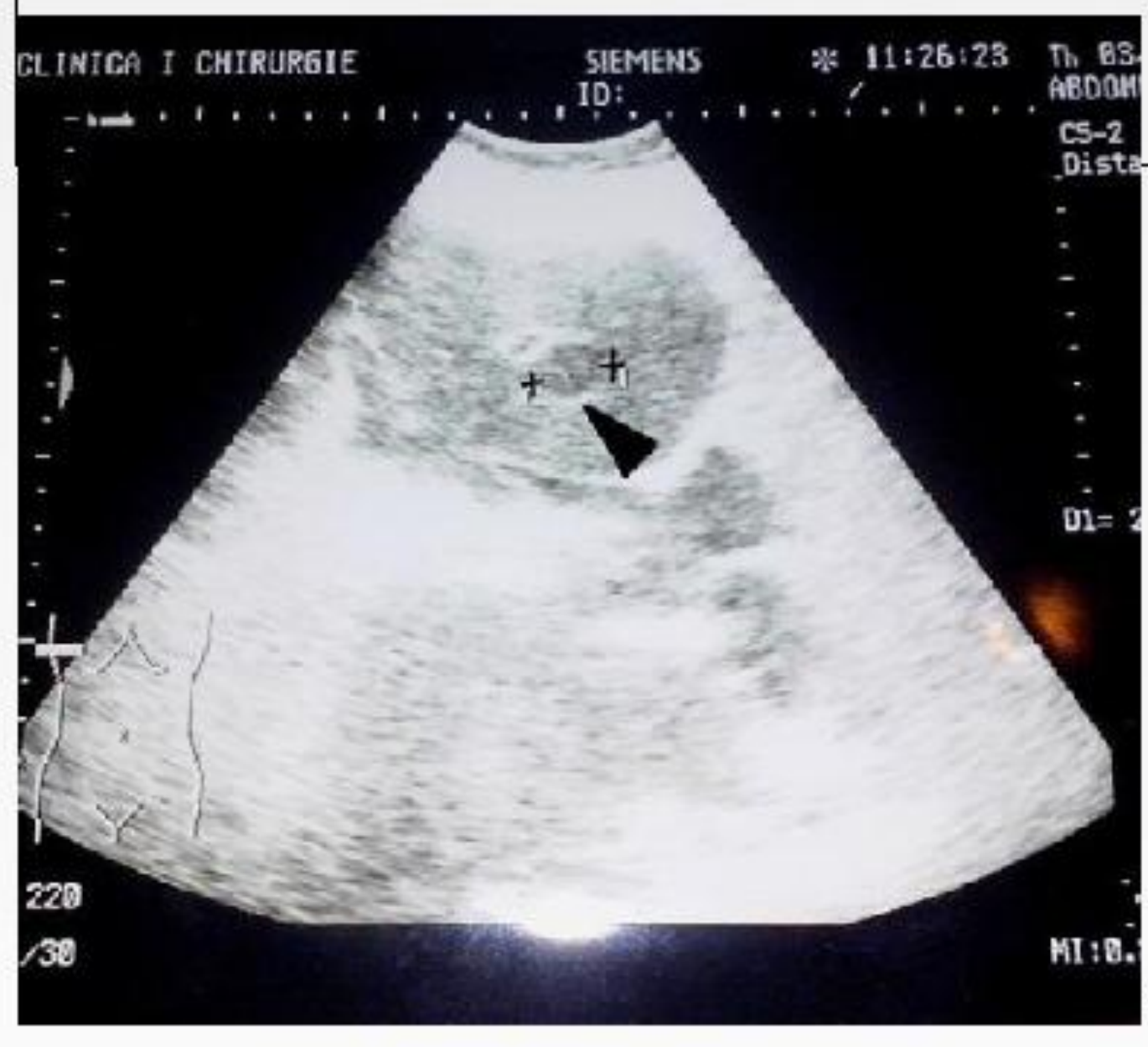

Figure 1 - Abdominal ultrasoun

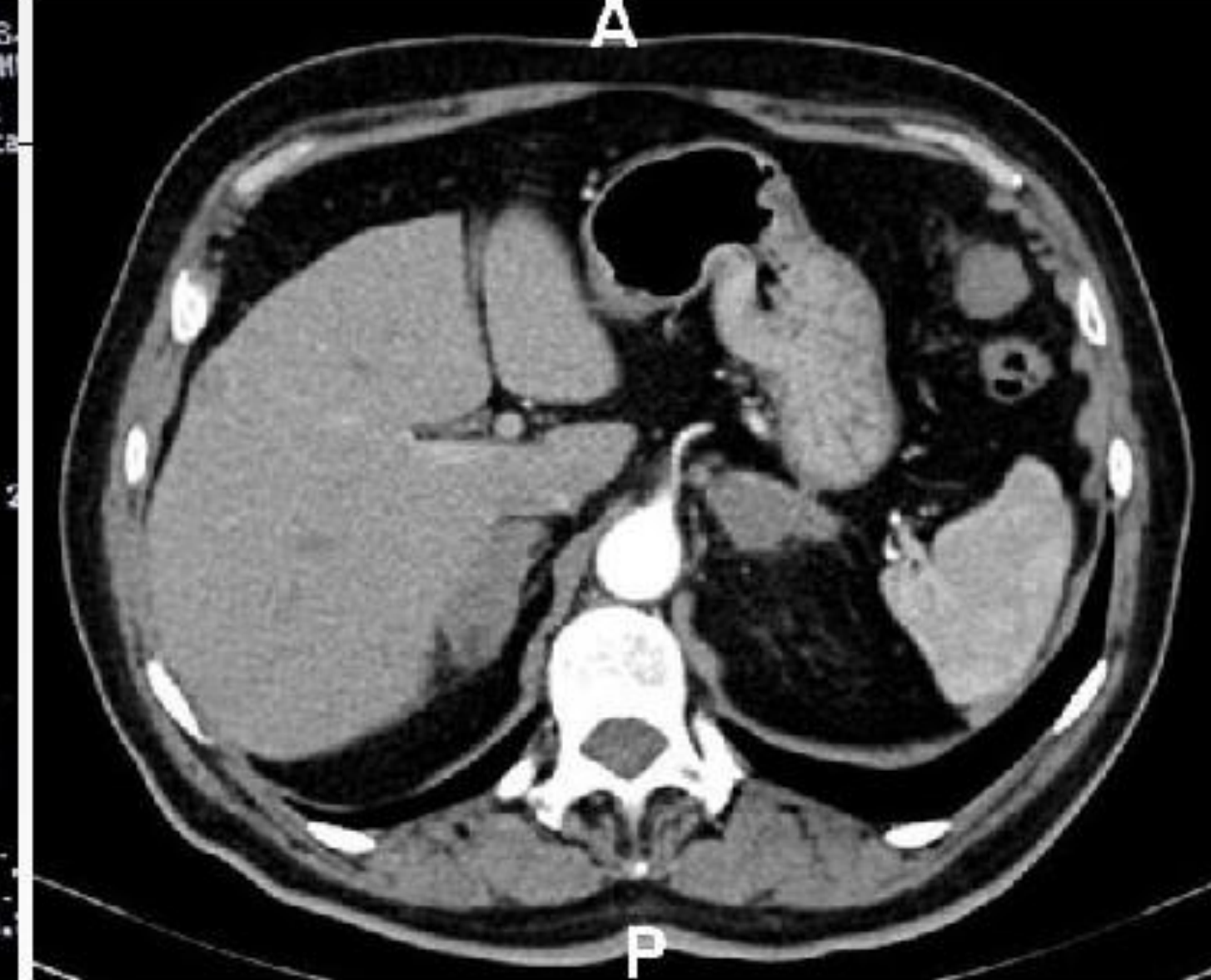

Figure 2 - Abdominal CT

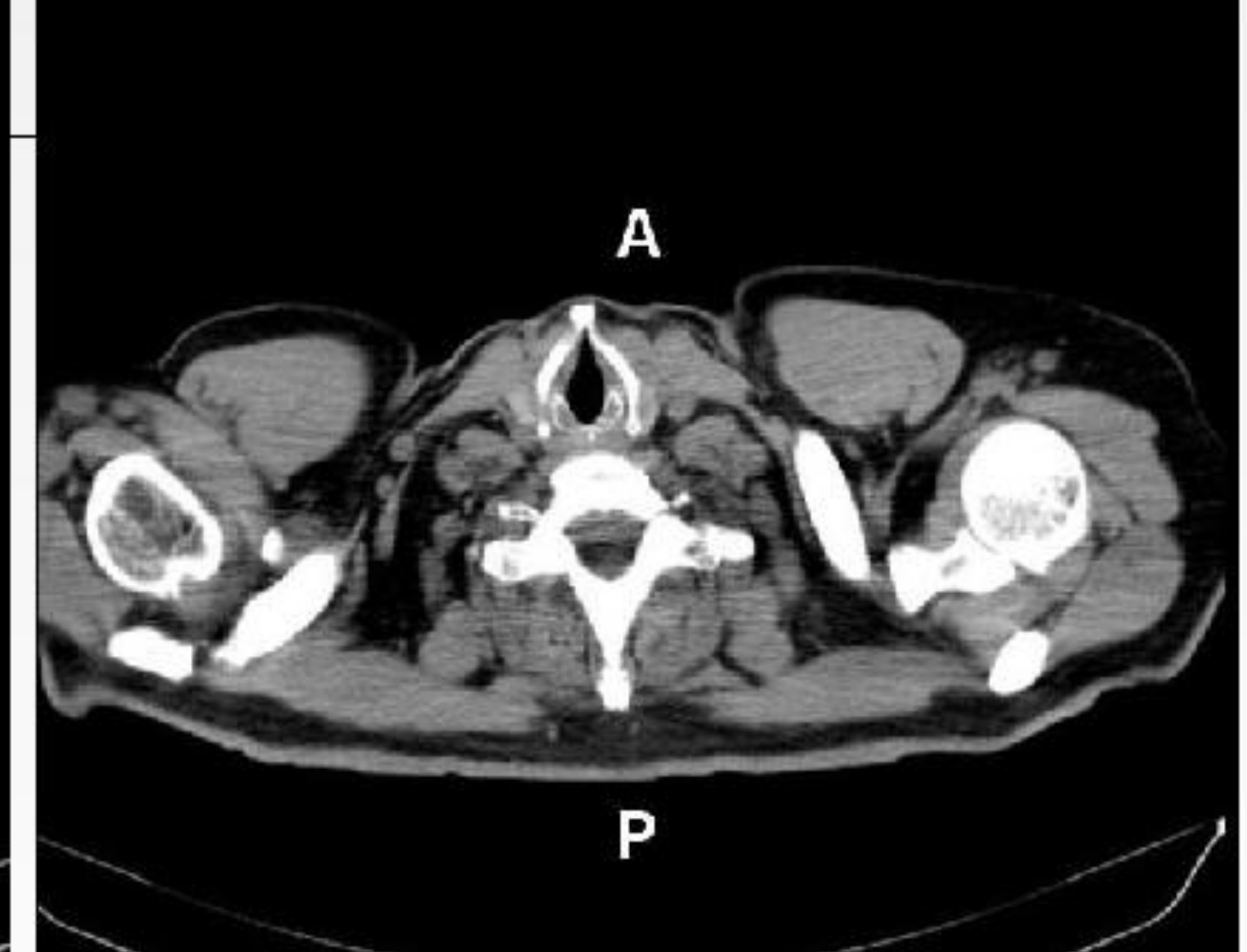

Figure 3 - Thotacic CT
Pacient V B, 57 years old

(heavy smoker and alcohol consumer)

In july 2014, after an intervention for Dupuytren retraction, he decompensated primary adrenal insuficiency

(anamnestic, confirmed at that time by the biological data).

Steroid substituion was started with a good initial evolution.

After 3 months he stopped the treatment and was hospitalized with adrenal crisis:

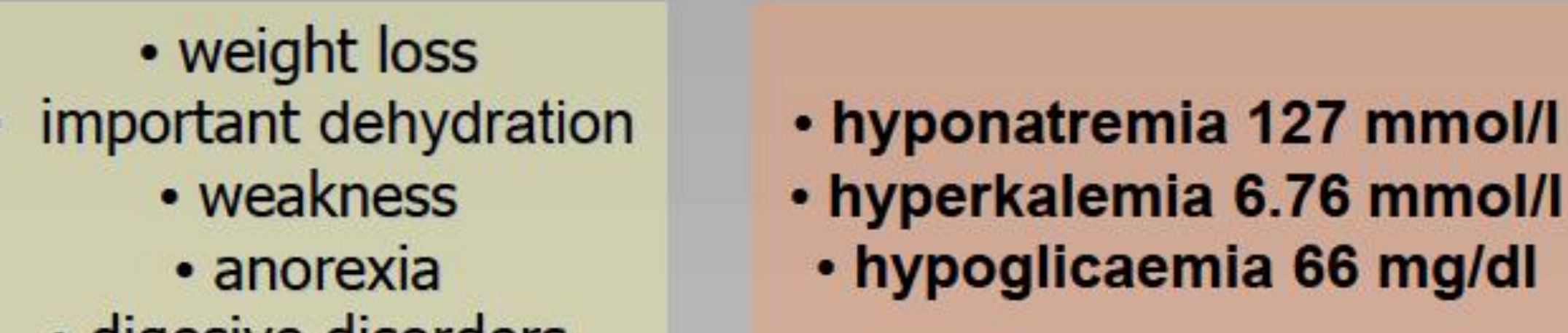

- digesive disorders

Intensive treatment ameliorated his status but the sudden discontinuation of cortisone replacemen therapy led to a new acute decompensation, hospitalized in the Endocrinology Clinic.

He presented with:- diffuse hyperpigmentation

Pancoast Tobias syndrome: enophthalmos, right eyelid ptosis, shoulder and right arm pain,

very low levels of cortisol $<1 \mathrm{ug} / \mathrm{dl}$ and normal ACTH levels $15.2 \mathrm{pg} / \mathrm{ml}$

* Abdominal ultrasound (fig. 4) revealed large left adrenal lesion (30/26 mm).

Corticosteroid replacement therapy ameliorated the status but the persistence of anorexia, asthenia

and inflammatory syndrome (ESR $115 \mathrm{~mm}$, CRP $3.59 \mathrm{mg} / \mathrm{dl}$, fibrinogen $380 \mathrm{mg} / \mathrm{dl}$ ) suggested a

severe underlying cause.

* Pulmonary radiography (figure 5) showed right apical lung nodule (35/45/50 mm) confirmed by thoraco-abdominal CT, which revealed a bilateral adrenal invasion.

* Pulmonary biopsy confirmed poorly differentiated mucosecretory adenocarcinoma
Microscopy:

- laterocervical adenocarcinoma

with high rate of multiplication
Immunohistochemistry:

- CK 7 and CK 5 positive

- chromogranin negative

TTF1 difuse positiv - Ki67 90\%
* Chemotherapy was proposed as the best option of treatment at this stage, refused by the patient.

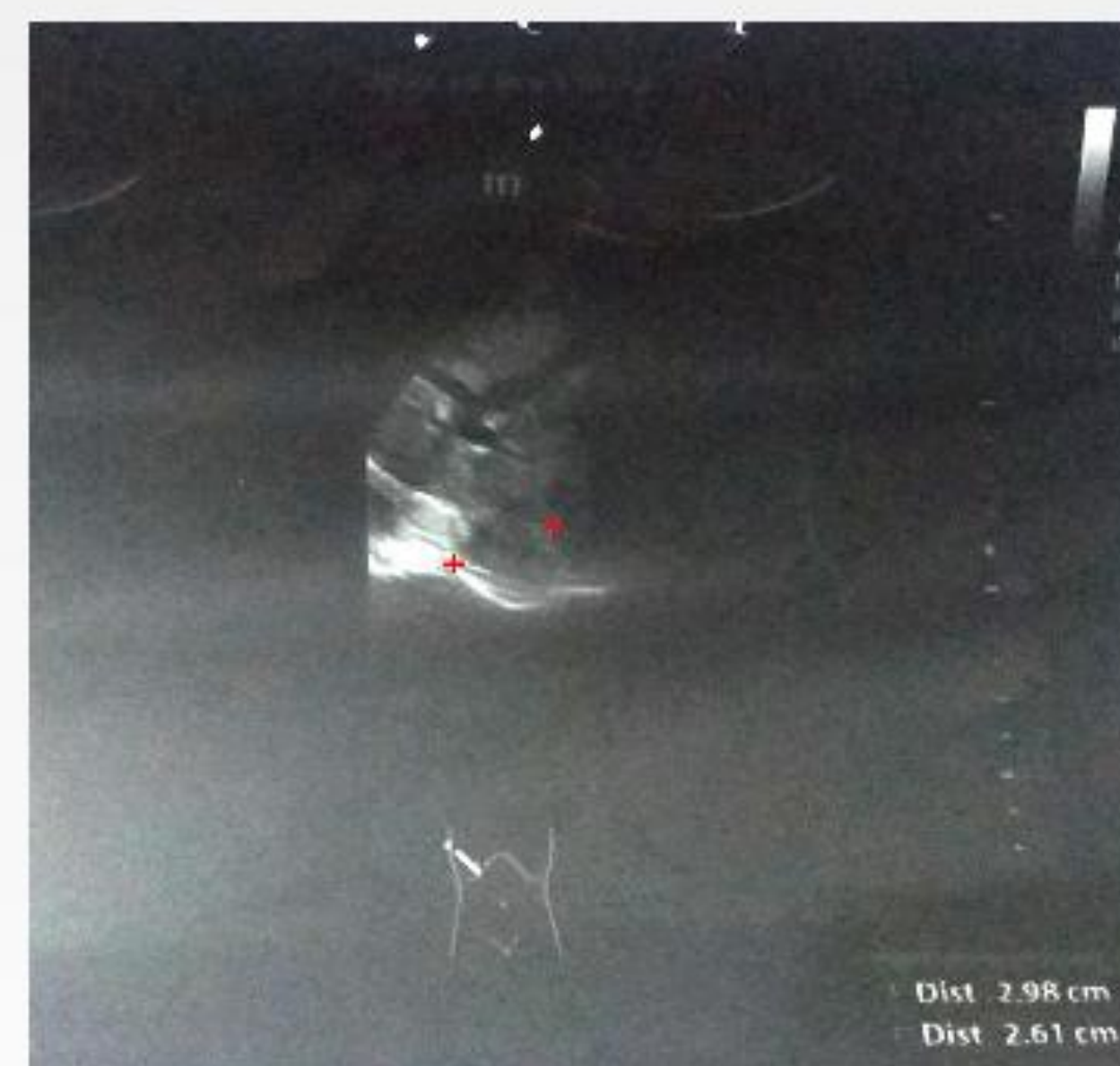

Figure 4 - Left drenal ultrasound
CONCLUSIONS

$\checkmark$ The frequency of adrenal metastasis of primary lung cancer increases with disease progression, from 10 to $40 \%$.

$\checkmark$ Although adrenal metastases are usually unilateral, bilateral adrenal metastases are seen in $10 \%$ of all lung cancer patients.

$\checkmark$ Clinical manifestations of adrenal insufficiency are significantly less frequent.

$\checkmark$ Patients with adrenal secondary lesions are typically asymptomatic, probably because a destruction of more than $90 \%$ of adrenal cortex is

needed for clinical symptoms

$1 \%$ may present with adrenal insufficiency as a first manifestation.

$\checkmark$ Adrenal crisis was, in our two cases, the first symptom of advanced pulmonary cancer, leading to its diagnostic and therapeutical solutions.

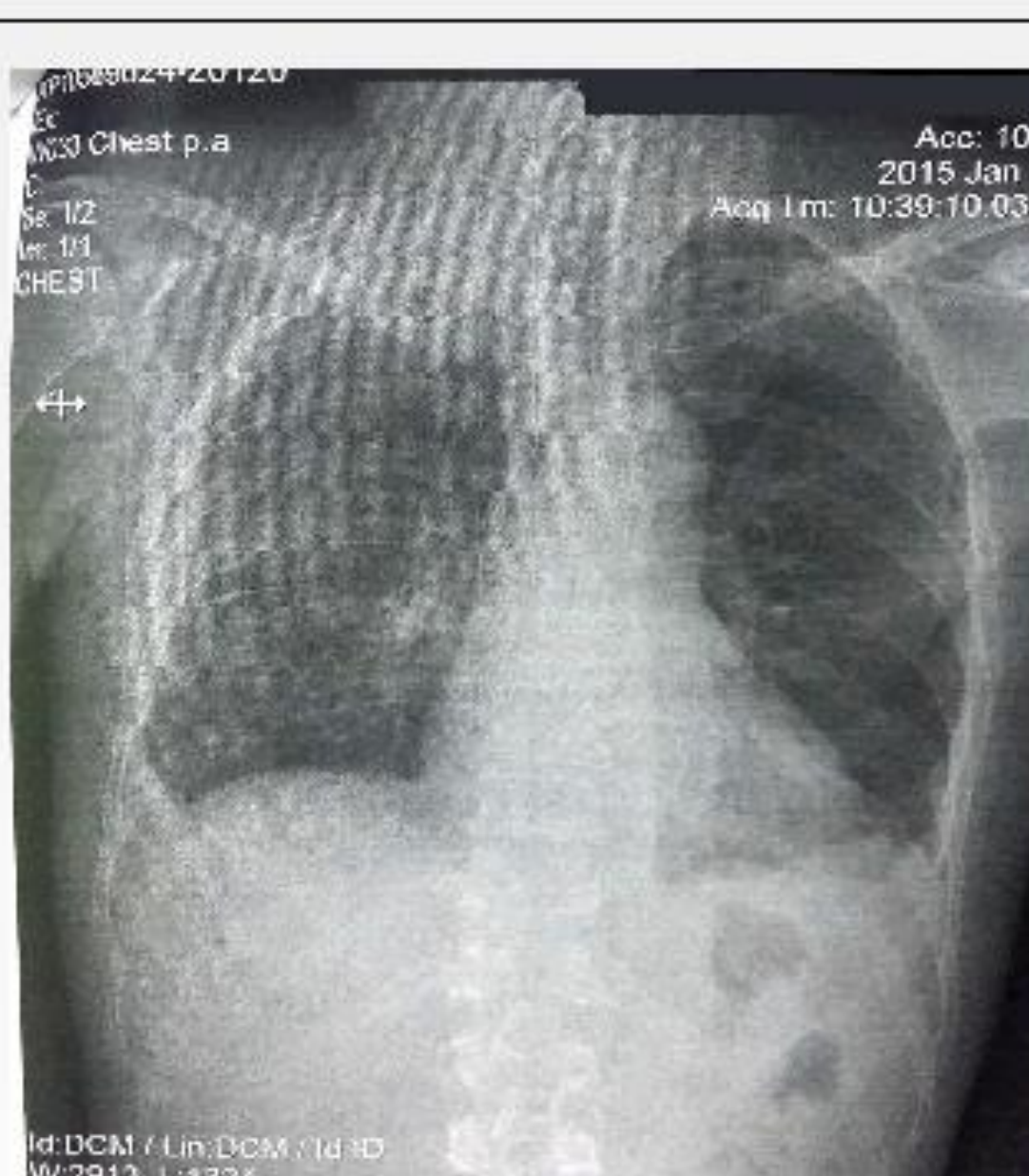

Figure 5- Thoracic radiography

\section{REFERENCES:}

